\title{
RELACIÓN QUE EXISTE ENTRE EL NIVEL DE CONOCIMIENTO Y LA APLICACIÓN DE PROTOCOLOS DE ASPIRACIÓN DE SECRECIONES POR TET EN LA UNIDAD DE CUIDADOS INTENSIVOS DEL HOSPITAL GUILLERMO ALMENARA IRIGOYEN, 2009
}

\author{
Orfelina ARPASI QUISPE 1 , Moraima ROCA BANCES ${ }^{2}$ y María VILLANUEVA SOLANO $^{3}$
}

\begin{abstract}
RESUMEN
Objetivo: Determinar la relación que existe entre el nivel de conocimiento y la aplicación del protocolo de aspiración de secreciones en la Unidad de Cuidados Intensivos del Hospital Nacional Guillermo Almenara Irigoyen. Metodología: El tipo de estudio es descriptivo de diseño correlacional transversal. Se aplicó una encuesta para determinar el nivel de conocimiento del protocolo de aspiración; y para valorar su aplicación por las enfermeras asistenciales de la Unidad de Cuidados Intensivos, se aplicó una guía de observación a un total de 43 enfermeras. La correlación estadística se realizó mediante la prueba Chi cuadrada. Resultados: De las enfermeras evaluadas, tanto en conocimiento y aplicación del protocolo (43), el 58.1\% tiene conocimiento entre bueno y excelente, mientras que el 53.5\% tiene un nivel de aplicación del protocolo de aspiración de secreciones malo. La correlación según la prueba Chi cuadrado muestra una significancia de 0.5. Conclusión: El nivel de conocimiento no se correlaciona con la aplicación del protocolo de aspiración de secreciones.
\end{abstract}

Palabras clave: Conocimiento, observación, aspiración de secreciones.

\begin{abstract}
Objective: To determine the relationship between the level of knowledge and application of the suctioning protocol of secretions in the Intensive Care Unit at the Guillermo Almenara Irigoyen General Hospital. Methodology: The type of study is descriptive, cross correlational. A survey was applied to determine the level of suctioning protocol knowledge and to assess their application by the nurses of the Intensive Care Unit. An observation guide was applied to a total of 43 nurses. The statistical correlation was performed through the chi square. Results: Of the nurses evaluated both in knowledge and application of the protocol (43) 58.1\% have knowledge between good and excellent, while $53.5 \%$ have a bad level in the application of the suctioning protocol of secretion. The correlation by chi-square test shows a significance of 0.5 . Conclusions: The level of knowledge does not correlate with the implementation of the suctioning protocol of secretions.
\end{abstract}

Key Words: Knowledge, observation, suctioning of secretions.

\footnotetext{
${ }^{1}$ Enfermera Jefe del Servicio de Enfermería del Hospital II: Clínica Geriátrica San Isidro Labrador - Essalud y especialista en administración y gestión en enfermería.

${ }^{2}$ Enfermera coordinadora del Hospital Nacional Guillermo Almenara Irigoyen - EsSalud y especialista en administración y gestión en enfermería.

${ }^{3}$ Enfermera asistencial del Hospital Nacional Guillermo Almenara Irigoyen - EsSalud y especialista en administración y gestión en enfermería.
} 


\section{INTRODUCCIÓN}

En Estados Unidos de América dos millones de pacientes por año presentan infecciones intrahospitalarias (INH), y la cuarta parte de ellas ocurren en terapia intensiva, dando como resultado 90,000 muertes por año generando un costo anual para el Hospital Epidemiológico de Virginia de 4.5 a 5.7 billones de dólares (Weinstein RA.-1998 y Jarvis WR. 2001). El 70\% de las INH de debe a bacterias de resistencia múltiple y el tratamiento suele ser limitado y difícil. La susceptibilidad para contraerla está determinada más por el uso de objetos externos (catéter, tubo orotraqueal, etc.) que por enfermedades de base (Burke, 2003). Estas infecciones son adquiridas en el hospital con un tiempo de internamiento mínimo de 72 horas, siendo la neumonía nosocomial responsable en un 10 a $15 \%$ de todas las infecciones adquiridas en el hospital, con un riesgo de adquisición aproximadamente de 8.6 por cada 1.000 admisiones (Saldar, 2001).

Las INH se definen como cualquier enfermedad microbiológica clínicamente reconocible que afecta al paciente como consecuencia de su admisión, permanencia y/o después de su egreso del hospital. Se reconoce que estas infecciones tienden a aumentar en la medida que se incorporan nuevos procedimientos invasivos, diagnosticándose durante la práctica médica (MINSA, 2004).

En el Perú, a nivel nacional, las infecciones respiratorias constituyen la tasa de morbilidad de $8.06 \%$ y la tasa de mortalidad de $18.6 \%$ (Apolinario, 2002). En algunos hospitales como el Hospital Nacional Hipólito Unanue en el área de Intermedios del Servicio de Medicina, las Infecciones Intrahospitalarias representan una tasa de morbilidad de $4.55 \%$ y mortalidad de $6.25 \%$, en el año 2000.

Por otro lado, el MINSA desde el 2001 hasta la fecha ha realizado cambios en el manejo temprano del paciente críticamente enfermo utilizando guías y consensos, creados en base a la luz de los nuevos conocimientos y observaciones del quehacer diario en las unidades de cuidados intensivos, los cuales han sido validados internacionalmente por grupo de expertos. (Coronado, 2006).

La Oficina de Inteligencia Sanitaria del Hospital Guillermo Almenara reportó en el 2008 que el 9.4\% de neumonías nosocomiales estaba asociada al ventilador mecánico.

El personal de enfermería es parte del equipo multidisciplinario y es responsable de las medidas preventivas durante la aplicación del procedimiento de aspiración de secreciones, sobre todo en pacientes intubados quienes presentan mayor riesgo de complicaciones. En este sentido, la enfermera debe contar con información suficiente sobre la técnica respectiva y la fundamentación científica de su proceder, así como la aplicación adecuada del protocolo de aspiración de secreciones, lo que permitirá prevenir las complicaciones anteriormente mencionadas (Jiménez, 2004).

En este sentido, las investigadoras, en su calidad de enfermeras asistenciales de la Unidad de Cuidados Intensivos del Hospital Nacional Guillermo Almenara, han observado que algunos pacientes presentan complicaciones como neumonías nosocomiales; asimismo, durante los turnos de trabajo han podido observar que algunas enfermeras con experiencia en el área de cuidados intensivos así como el personal de reciente ingreso, al realizar la aspiración de secreciones de un paciente a otro no se lavan las manos, muchas veces también aspiran al paciente estando en posición de cúbito dorsal sin levantar la cabecera, lo que podría poner en riesgo al paciente internado en este servicio. Además, se ha observado que algunas enfermeras omiten la valoración del paciente auscultando ambos campos pulmonares antes de realizar el procedimiento de aspiración de secreciones.

Frente a esta realidad, el presente estudio tiene como objetivo de investigación determinar la relación que existe entre el nivel de conocimiento y la aplicación de protocolo de aspiración de secreciones en la Unidad de Cuidados Intensivos del Hospital Nacional Guillermo Almenara Irigoyen.

\section{MATERIAL Y MÉTODOS}

El presente estudio de investigación es descriptivo de diseño correlacional transversal. Se consideró a todo el personal de enfermería del servicio de Cuidados Intensivos del Hospital Nacional Guillermo Almenara Irigoyen - EsSalud, que estuvieron laborando durante el mes de abril de 2009; se excluyeron a las enfermeras que se encontraron de vacaciones, descanso médico y a quienes se negaron a participar. El total de las enfermeras que intervinieron en el estudio fueron 43.

La recolección de datos para la variable: nivel de conocimiento del personal de enfermería, se realizó mediante un cuestionario de 28 preguntas. Para determinar la práctica de la aspiración de secreciones a las enfermeras de la UCI se evaluó a través de una guía de observación de 20 ítemes.

Para ambos casos se aplicó la siguiente escala:
$00-10=$ Malo
$14-16=$ Bueno
$11-13=$ Regular
$17-20=$ Excelente 
Ambos instrumentos fueron elaborados por las investigadoras mediante la operacionalización de las variables y su respectiva validación por juicio de expertos.

El cuestionario aplicado al personal fue de forma anónima. Obtenida la recolección de datos se realizó el análisis correspondiente con la ayuda del software SPSS para Windows.

Se aplicó como estadístico la prueba de Chi cuadrado para relacionar el nivel de conocimiento y la aplicación del protocolo.

\section{RESULTADOS}

Tabla 1. Nivel de conocimiento de aspiración de secreciones de las enfermeras del servicio de cuidados intensivos del Hospital Nacional Guillermo Almenara Irigoyen, 2009.

\begin{tabular}{|l|c|c|}
\hline NIVEL & Frecuencia & Porcentaje \\
\hline EXCELENTE & 4 & 9,3 \\
\hline BUENO & 21 & 48,8 \\
\hline REGULAR & 17 & 39,5 \\
\hline MALO & 1 & 2,3 \\
\hline TOTAL & 43 & 100,0 \\
\hline
\end{tabular}

Fuente: Encuesta de nivel de conocimiento, aplicada en la Unidad de Cuidados Intensivos del Hospital Guillermo Almenara Irigoyen ESSALUD, Abril 2009

En la tabla 1 se muestra el nivel de conocimiento de las enfermeras de la UCI del Hospital Nacional Guillermo Almenara Irigoyen sobre el protocolo de aspiración de secreciones, en donde se observa que el 48,8 \% de ellas presenta un nivel de conocimiento bueno, el 39\% presenta un nivel regular, el 9\%, excelente y sólo el $2.3 \%$, malo.

Tabla 2. Aplicación del protocolo de aspiración de secreciones de las enfermeras del servicio de cuidados intensivos del Hospital Nacional Guillermo Almenara Irigoyen, 2009.

\begin{tabular}{|l|c|c|}
\hline NIVEL & Frecuencia & Porcentaje \\
\hline EXCELENTE & 1 & 2,3 \\
\hline BUENO & 2 & 4,7 \\
\hline REGULAR & 17 & 39,5 \\
\hline MALO & 23 & 53,5 \\
\hline TOTAL & 43 & 100,0 \\
\hline
\end{tabular}

Fuente: Guía de Observación, aplicada en la Unidad de Cuidados Intensivos del Hospital Guillermo Almenara Irigoyen - ESSALUD, abril 2009

En la tabla 2 se observa que el $53.5 \%$ de las enfermeras tiene un nivel malo de aplicación del protocolo de aspiración de secreciones, el 39\% presenta un nivel regular, el 4\% bueno y sólo el 2\% presenta un nivel excelente.

Tabla 3. Pruebas de Chicuadrado entre nivel de conocimiento y aplicación de protocolo de aspiración de secreciones de las enfermeras de la Unidad de Cuidados Intensivos del Hospital Guillermo Almenara Irigoyen, 2009.

\begin{tabular}{|l|c|l|c|}
\hline & Valor & gl. & $\begin{array}{c}\text { Sig. asintótica } \\
\text { (bilateral) }\end{array}$ \\
\hline Chi-cuadrado de Pearson & $8.318(\mathrm{a})$ & 9 & 0.502 \\
\hline Razón de verosimilitud & 9.808 & 9 & 0.366 \\
\hline Asociación lineal por lineal & 0.194 & 1 & 0.659 \\
\hline $\mathrm{N}^{\circ}$ de casos válidos & 43 & & \\
\hline TOTAL & 43 & & 100,0 \\
\hline
\end{tabular}

Fuente: Encuesta de nivel de Conocimiento, aplicada en la Unidad de Cuidados Intensivos del Hospital Guillermo Almenara Irigoyen - ESSALUD, abril 2009

En la tabla 3, muestra que el valor de significancia según la prueba Chicuadrado es mayor a $0.05(\mathrm{P}=0.00>0.05)$; por lo tanto, no hay correlación entre el nivel de conocimiento y la aplicación del protocolo de aspiración de secreciones. 


\section{DISCUSIÓN}

En el presente estudio las investigadoras encontraron que el nivel de conocimiento de todas las enfermeras observadas de la UCI del Hospital nacional Guillermo Almenara Irigoyen sobre el protocolo de aspiración de secreciones es bueno en un 48,8\%, además se encontró que el nivel de aplicación del protocolo de aspiración de secreciones es malo en un $53.5 \%$ y solamente un $7 \%$ tiene un nivel de aplicación entre bueno y excelente.

Rodríguez (1994), refiere que el conocimiento es un conjunto de datos sobre hechos, realidades y de información almacenada a través de la experiencia o el aprendizaje. Entendiendo que la aspiración de secreciones es una técnica cuyo propósito es el mantener las vías aéreas permeables retirando del árbol bronquial las secreciones que un paciente no puede eliminar espontáneamente, evitando así infecciones nosocomiales, debemos de tener en cuenta que el conocimiento y la práctica forman una sola unidad; es decir, que la aplicación del protocolo es el resultado del conocimiento obtenido coadyuvando a un cuidado de calidad, tal como lo menciona Castro (1998).

Contradictoriamente, al realizar la prueba de Chicuadrado muestra que no hay correlación entre el nivel de conocimiento y la aplicación del protocolo de aspiración de secreciones de las enfermeras de la Unidad de Cuidados Intensivos del Hospital Guillermo Almenara.

Asimismo, esta técnica debe ser aplicada permanentemente por el personal de enfermería, de acuerdo a las pautas que indica el protocolo del servicio. Apolinario (2002) en un estudio realizado en el Hospital Hipólito Unanue refiere que un $76 \%$ de las enfermeras conocimiento bueno sobre el protocolo de aspiración de secreciones y un $73 \%$ de las enfermeras tienen una buena práctica del protocolo.
Sin embargo, los resultados obtenidos en la presente investigación muestran que de todas las enfermeras, sujetas a investigación, solamente un 7\% tiene un nivel de aplicación entre bueno y excelente. Teniendo en cuenta que la enfermera desempeña un rol importante en la Unidad de Cuidados Intensivos, y debe actuar oportunamente con el paciente para prevenir complicaciones nosocomiales, podría haber factores que estarían afectando la práctica adecuada de las enfermeras sobre la aspiración de secreciones.

Por todo lo mencionado se cree pertinente plantear las siguientes recomendaciones:

1. Difusión y capacitación al personal de enfermería sobre el protocolo de aspiración de secreciones.

2. Hacer efectivo un programa de inducción al personal de reciente ingreso al servicio.

3. Realizar otro estudio de investigación para determinar los factores que influyen en el conocimiento y aplicación en el procedimiento de aspiración de secreciones.

\section{CONCLUSIONES}

1. El nivel de conocimiento de las enfermeras sobre aspiración de secreciones no se correlacionan con la aplicación del protocolo.

2. El nivel de conocimiento de la mayoría de las enfermeras sobre aspiración de secreciones es bueno; sin embargo, el nivel de aplicación del protocolo de aspiración de secreciones de la mayoría de las enfermeras es malo. 


\section{REFERENCIAS BIBLIOGRÁFICAS}

1. Apolinario Mendivil, Roxana Emilia. Conocimientos y prácticas que tienen las enfermeras sobre la aspiración de secreciones en pacientes intubados en la unidad de cuidados intermedios del Hospital Nacional Hipolito Unanue. Lima; 2002

2. Cañari De La Cruz, Ruth. Conocimiento que tiene la enfermera sobre anotaciones de enfermería y los factores personales e institucionales que limita su elaboración en los hospitales. Perú: Almenara; 1998.

3. Durand de Villalobos, María. Boletín Indagación filosófica y la práctica de enfermería. Colombia: Universidad Nacional; 1999. p.26.

4. Esteve J, Mitjans J. Enfermería. Técnicas clínicas. Madrid: McGraww-Hill Interamericana; 2002.

5. Hernández Rodríguez José, Díaz Hernández Máximo. Guía de intervención rápida de enfermería en cuidados intensivo. Colombia: Distribuna; 2008.

6. Jiménez Gómez, María Antonia. Cómo cuidar al paciente con soporte mecánico ventilatorio. Bogotá: Unibiblos; 2004.
7. Lillian Sholtis Brunner. Tratado de enfermería. México: Editorial Interamericana, S.A.; 1986.

8. OBCIT Rodríguez, Francisco. Introducción a la Metodología de la investigación; 2002. p. 3.

9. Regina Laselva Claudia, Moura Junior Denis. Terapia intensiva en enfermería. Colombia: Distribuna; 2008.

10. Rodriguez Francisco. Introducción a la Metodología de la investigación social. Editorial política; 1994. p.15.

11. Rueda Rojas, Norma. Nivel de conocimiento y aplicación de las notas de enfermería de sala de operación del Hospital Alberto Sabogal Sologuren. Callao; 2007.

12. Ruiz J, Martín MC, García-Penche R. Procedimientos y Técnicas de Enfermería. ROL; 2006.

13. Urden, Linda. Cuidados Intensivos en Enfermería. Madrid: Iberoamericana; 1998.

14.Urden Linda, Lough Mary, Stacy Kathleen. Cuidados Intensivos en Enfermería. España: Harcourt Brace; 1998. 